\title{
AN ARTIFICIAL NEURAL NETWORK MODEL FOR PREDICTING THE BEHAVIOUR OF SEMI-RIGID JOINTS IN FIRE
}

\author{
K.S. Al-Jabri ${ }^{1, *}$, S.M. Al-Alawi ${ }^{2}$, A.H. Al-Saidy ${ }^{1}$ and A.S. Alnuaimi ${ }^{1}$ \\ ${ }^{1}$ Department of Civil and Architectural Engineering, College of Engineering, Sultan Qaboos University, Oman \\ ${ }^{2}$ Department of Electrical and Computer Engineering, College of Engineering, Sultan Qaboos University, Oman \\ *(Corresponding author: E-mail: aljabri@squ.edu.om)
}

Received: 9 April 2008; Revised: 14 July 2008; Accepted: 29 July 2008

\begin{abstract}
This paper presents an artificial neural networking (ANN) model developed to predict the behaviour of semi-rigid bare-steel joints at elevated temperature. Data for three flush end-plate and one flexible end-plate joints were considered. Sixteen parameters which included geometry of the joint's components, material properties of the joint, joint's temperature and the applied moment were used as the input variables for the model whilst the joint's rotation was the main output parameter. Data from experimental fire tests were used for training and testing the model. In total, fifteen different test results were evaluated with 331 and 61 cases were used for training and testing the developed model, respectively. The model predicted values were compared with actual test results. The results obtained indicated that the model can predict the moment-rotation behaviour in fire with very high accuracy. The coefficients of determination $\left(\mathrm{R}^{2}\right)$ for training and validation of the model were 0.964 and 0.956 , respectively.
\end{abstract}

Keywords: Bare-steel, flush end-plate, flexible end-plate, semi-rigid joints, artificial neural network, fire, elevated temperature, rotation

\section{INTRODUCTION}

Steel loses both its strength and stiffness when subjected to fire. Fire tests on steel structures have shown that the temperature within the joints is lower compared to connecting steel members. This is attributed to the additional material around joints (column, end-plate, concrete slab, etc.) which significantly reduces the temperatures within the connections compared to those at the centre of supported beams. The experimental results on the behaviour of steel connections under fire conditions are relatively recent and limited, partly because of the high cost of the fire tests and the limitations on the size of furnaces used. Only limited joint tests have been performed and they were concentrated on obtaining the moment-rotation relationships of isolated joints (Al-Jabri et al. [1]). Therefore, experimental fire joint tests are not anticipated to be performed on many connection types with various end conditions. It is well known that even nominally 'simple' connections can resist significant moments at large rotation. At the severe deformation of structural members in fire, moments are transferred through the joints to the adjacent members, and hence, they may have a beneficial effect on the survival time of members.

Accurate prediction of the structural behaviour of steel beam-to-column connections, by estimating the local deformations and induced stresses, is necessary to assess the capacity of the connections and prevent their failure. Numerical modeling presents, in principle, an alternative way to predict the response of structural steel joints in fire. Artificial neural network (ANN) modeling is an artificial intelligence-based technique that emulates the human ability to learn from the past experience and derive quick solutions to new problems. The developed ANN-based prediction model can be used by structural engineers to predict the elevated temperature behaviour of similar structural members. 
Few ANN models have been developed to predict the connection behaviour at ambient temperature. Stavroulakis and Abdalla [2] and Stavroulakis et al. [3] described a neural network approach for identification and classification of semi-rigid connections in steel structures. The moment-rotation law of the connection was obtained from experimental results by the use of a neural network based on the perceptron model. Then the elastic plastic analysis problem was formulated for the given moment-rotation law as a quadratic programming problem and solved by a neural network based on the Hopfield model. The bi-linear moment-rotation characteristics for minor axis steel connections were predicted by Anderson et al. [4] using ANN. The results from the model were compared with experimental tests in which significant parameters have been varied. The results were found to provide approximations to the experimental response that are satisfactory for use in structural engineering design. Recently, a neural network model was proposed by Demirtas et al. [5] to obtain nonlinear moment-rotation curves for semi-rigid connections. The resulting model was then integrated into a non-linear frame analysis program to obtain nodal displacements and corresponding frame element forces. Results from the analysis were compared with experimental results for a two-storey one-bay frame with semi-rigid connections. Al-Khaleefi et al. [6] reported the possibility of predicting the behaviour of structural members in fire after investigating the fire resistance of concrete filled tubular steel columns using neural networks. The fire resistance of thirty-five hollow steel columns filled with plain concrete was predicted. Results were in good agreement with results obtained from experimental column tests.

This paper presents a new artificial network model developed to predict the behaviour of semi-rigid bare-stee1 unstiffened joints under fire conditions. The developed ANN can be used for similar connections under fire by observing various factors influencing the connection's behaviour such as: (a) geometrical factors (i.e., member sizes), (b) material factors (i.e., strength and stiffness), and (c) loading conditions (i.e., static and fire). The researcher is required to provide the magnitude of these influencing factors as inputs to the neural network and the network will predict the behaviour of the joint based on the combined effects of these factors. This model can be used to predict the rotational capacity of the connection and the contribution of individual components of the connection as well as the contribution of the loading to the overall connection's behaviour without conducting costly fire tests.

\section{ARTIFICIAL NEURAL NETWORKS (ANNs)}

ANNs are computer programs that are trained in order to recognize both linear and nonlinear relationships among the input and the output variables in a given data set. In general, ANN applications in engineering have received wide acceptance. The popularity and acceptance of this technique stems from ANN features that are particularly attractive for data analysis. These features include handling of fragmented and noisy data; speed inherent to parallel distributed architectures, generalization capability over new data, ability to effectively incorporate a large number of input parameters, and its capability of modeling nonlinear systems.

One of the most common and frequently used ANN paradigm is the Back-propagation paradigm. This supervised learning method was developed by Rumelhart [7] based on the generalization of the least mean square error (LMS) algorithm. The Back-propagation algorithm uses gradient descent search technique to minimize a cost function equal to the mean square difference between the desired and the actual net output. The network is trained by selecting small random weights and internal threshold and then presenting all training data repeatedly by using supervised training technique. The weights are changed till the network reach the desired error level or the cost function is reduced to an acceptable value. 
The major building block for any ANN architecture is the processing element or neuron. These neurons are located in one of three types of layers: the input layer (weights), the hidden (bias) layer, or the output (an activation function) layer. The input neurons receive data from the outside environment, the hidden neurons receive signals from all of the neurons in the preceding layer, and the output neurons send information back to the external environment. The neurons in the input layer receive the input signals representing the input parameters which describe the system modelled such as geometrical and material properties of the joint, loading, temperature, etc. The output layer, on the other hand, consists of output neuron representing the predicted results obtained from the ANN modelling such as joint's rotation, internal forces of the joint's components, etc. Between the input and output layers, generally, there is one or more hidden layer. These neurons are connected together by a line of communication called connection. Stanley [8] indicated that the way in which the neurons are connected to each other in a network typology has a great effect on the operation and performance of the network. ANN models come in a variety of typologies or paradigms. Simpson [9] provides a coherent description of 27 different popular ANN paradigms and presents comparative analyses, applications, and implementations of these paradigms.

In the back propagation (BP) architecture, shown in Figure 1, each element or neuron receives input from the real-world environment or from other processing elements, processes this input, and produces a specific output. Generally, many of these processing elements perform their operations at the same time. This parallelism is a unique feature of the ANN that distinguishes it from the serial processing that is usually performed by conventional computer systems. Each neuron has a straightforward assignment. Input coming to the neuron is associated with a weight indicating its strength. In the neuron, the values of the input are multiplied by the corresponding weights and all products are added to obtain a net value $\left(\right.$ net $\left._{i}\right)$. After summation, the net input of the neurons is combined with the previous state of the neurons to produce a new activation value. Whether the neurons fire or not will depend on the magnitude of this value. For example, each neuron receives inputs $x_{1}, x_{2}, \ldots \ldots . . x_{n}$, attached with a weight $w_{i}$ which shows the connection strength for that input for each connection. Each neuron is then multiplied by the corresponding weight of the neuron connection. A bias $b_{i}$ can be defined as a type of connection weight with a constant nonzero value added to the summation of inputs and corresponding weights $u$ as expressed in Eq. 1 .

$$
u_{i}=\sum_{j=1}^{H} w_{i j} x_{j}+b_{i}
$$

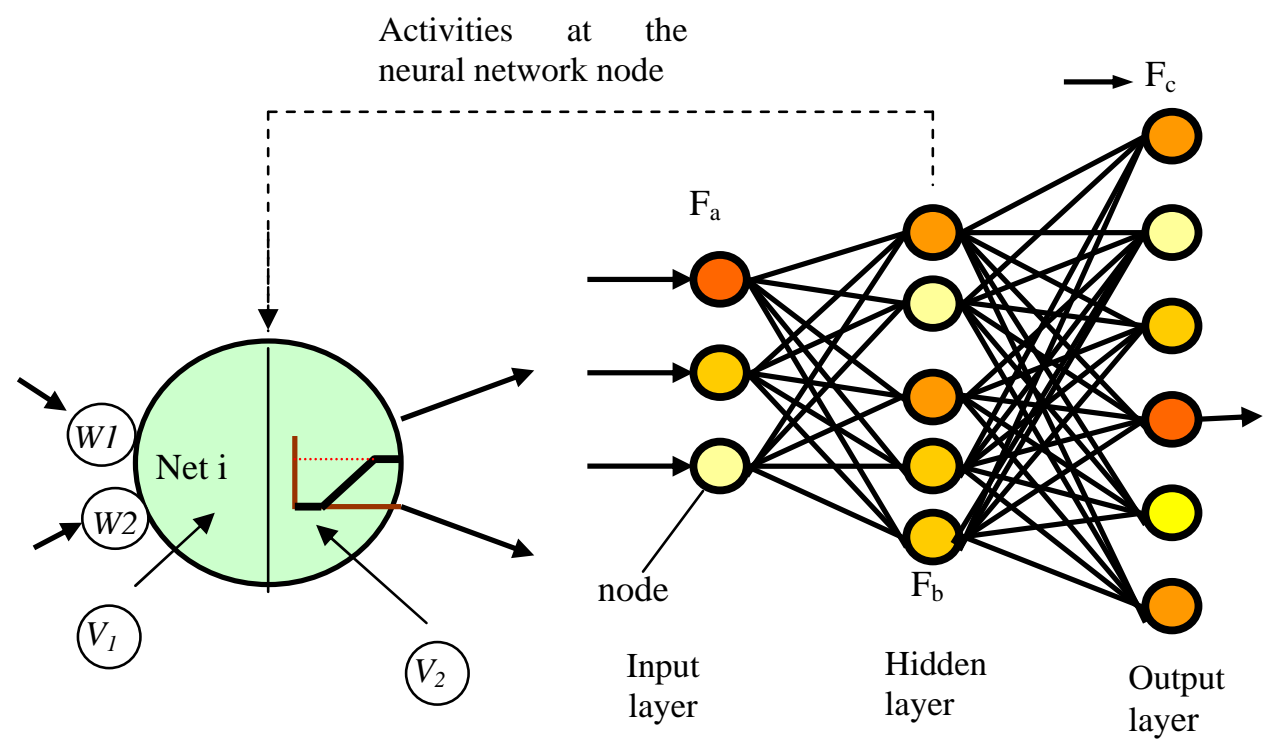

Figure 1. Typical Back-Propagation Architecture 
The activation is then passed through an output or a transfer function $\left(\mathrm{fu}_{\mathrm{i}}\right)$ that generates the actual neuron output. The transfer function modifies the value of the output signal. $f\left(\mathrm{u}_{\mathrm{i}}\right)$ yielding a value called the unit's “activation”, given in Eq. 2.

$Y_{i}=f(u i)$

This function can be either a simple threshold function that only produces output if the combined input is greater than the threshold value, or it can be a continuous function that changes the output based on the strength of the combined input. Activation functions serve to introduce nonlinearity into neural networks, which makes ANNs so powerful (Guzelbey et al. [10-11]). Typical transfer functions employed in building ANN applications include a linear threshold transfer function, step function, sigmoid function, and others. In this work a sigmoid function was used to develop the ANN Model.

Another important network design variable is the learning rate coefficient which represents the degree by which the weights are changed when two neuron are excited. Each time a pattern is presented to the network, the weights leading to a neuron are modified slightly during learning in the direction required to produce a smaller error at the outputs the next time the same pattern is presented. The amount of weight modification is proportional to the learning rate. The value of the coefficient ranges between 0.0 to 1.0 , where a value closer to 1 indicates significant modification in weight while a value closer to 0 indicates little modification (Al-Khaleefi et al. [6]).

\subsection{ANN Training and Testing}

The first and the most critical step in developing an effective ANN model is input and output definition and data preparation. This includes identifying variables of interest, gathering the relevant data and inspecting them for possible errors, missing values, and outliers. Data accuracy is vital for the development of an efficient model that can provide accurate prediction. If incorrect or erroneous data are fed to the model, this will result in incorrect prediction. As the saying goes, "garbage in, garbage out".

Once the ANN model architecture is defined, data are collected and fed to the model. The network is then trained to recognize the relationships between the input and output parameters. The BP algorithm uses the supervised training technique. In this technique, the interlayer connection weights and the processing elements' thresholds are first initialized to small random values. The network is then presented with a set of training patterns, each consisting of an example of the problem to be solved (the input) and the desired solution to this problem (the output). These training patterns are presented repeatedly to the ANN model and the error between actual and predicted results is calculated. Weights are then adjusted by small amounts that are dictated by the General Delta Rule (Rumelhart and McClelland [7]). This adjustment is performed after each completed iteration whenever the network's Computed output is different from the desired output. This process continues until weights converge to the desired error level or the output reaches an acceptable level.

The ANN model can sometimes learn something different than the relationships in the data. It also can memorize the data or part of this data without learning the relationships between variables or trends in the data. Hence, to insure network accuracy and the generalization capability, the network must be tested on a continuous basis and should be monitored during the training and testing operations. The testing operation is performed by passing a separate testing set to the trained ANN model and recording the results. These results are compared to actual results. The trained model is assumed to be successful if the model gives good results for that test set. To 
insure that ANN models provide correct prediction or classifications, the prediction results produced by ANN models can be validated against expert predictions for the same cases or it can be validated against the results of other computer programs or experimental tests.

\section{KNOWLEDGE REPRESENTATION}

The procedure adopted to model the rotational behaviour of semi-rigid joints in fire consists of the following steps:

1. Identification of the major factors that influence the behaviour of semi-rigid joints in fire.

2. Collection of a set of experimental cases with values for these identified factors along with the respective joint's rotation.

3. Coding of the laboratory test cases and corresponding value of rotation obtained based on experiments.

4. Development of ANN model, from the coded cases, which is capable of predicting the rotation of other similar joints in fire.

5. Compare results generated by the model with the corresponding experimental results and identifying the contribution of each input parameter on the overall behaviour of joint at high temperature.

\section{GEOMETRY OF THE JOINTS}

The cruciform bolted beam-to-column steel joints, tested experimentally by Leston-Jones [12] and Al-Jabri et al. [1], were considered. Two of the joints (Fire1 and Fire2) have the same member sizes but different end-plate thicknesses whilst the third joint (Fire3) has larger member sizes. The first two joints consist of two 254x102UB22 beams connected to a 152x152UC23 column using six M16 bolts and $8 \mathrm{~mm}$ (Figure 2) and $12 \mathrm{~mm}$ thick flush end-plates for Fires1 and 2, respectively. The third joint (Fire3) comprised a pair of 356 x 171UB51 beams connected to a 254 x 254UC89 column by $10 \mathrm{~mm}$ thick flush end-plates with eight M20 Grade 8.8 bolts (Figure 3). The fourth joint (Fire4) is a flexible end-plate joint with beam and column sizes similar to Fire3 but the end-plates dimension is different as shown in Figure 4. In total fifteen elevated temperature tests were modelled with 331 and 61 cases were used for training and testing the proposed model, respectively. Table 1 shows the loading level for each test.
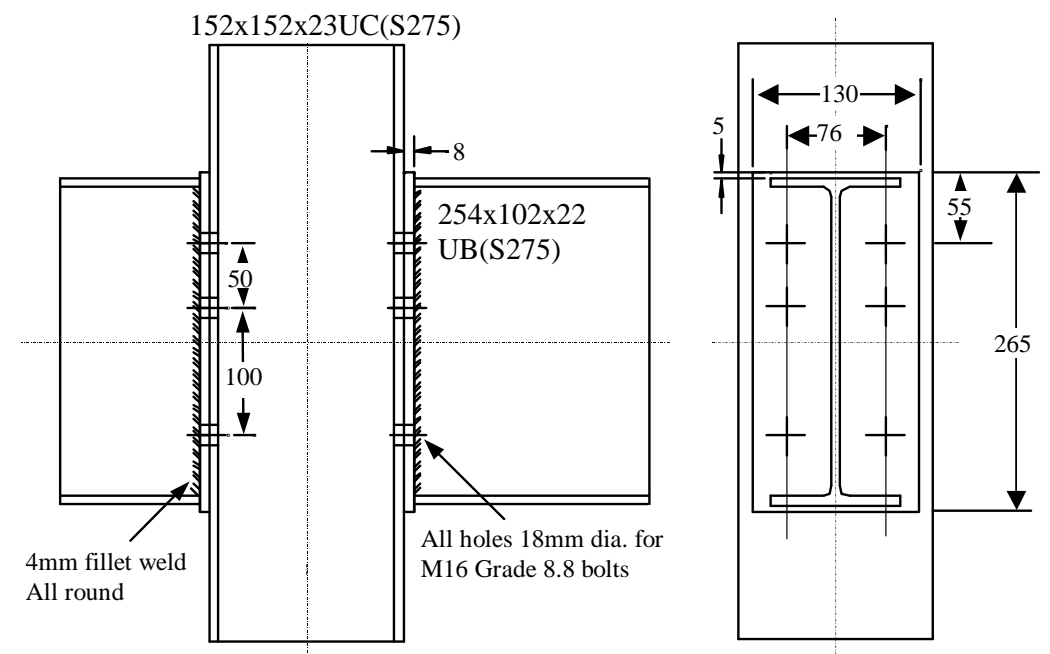

Figure 2. Flush End-Plate Joint Detail for Fire1 Tests (FR1) 

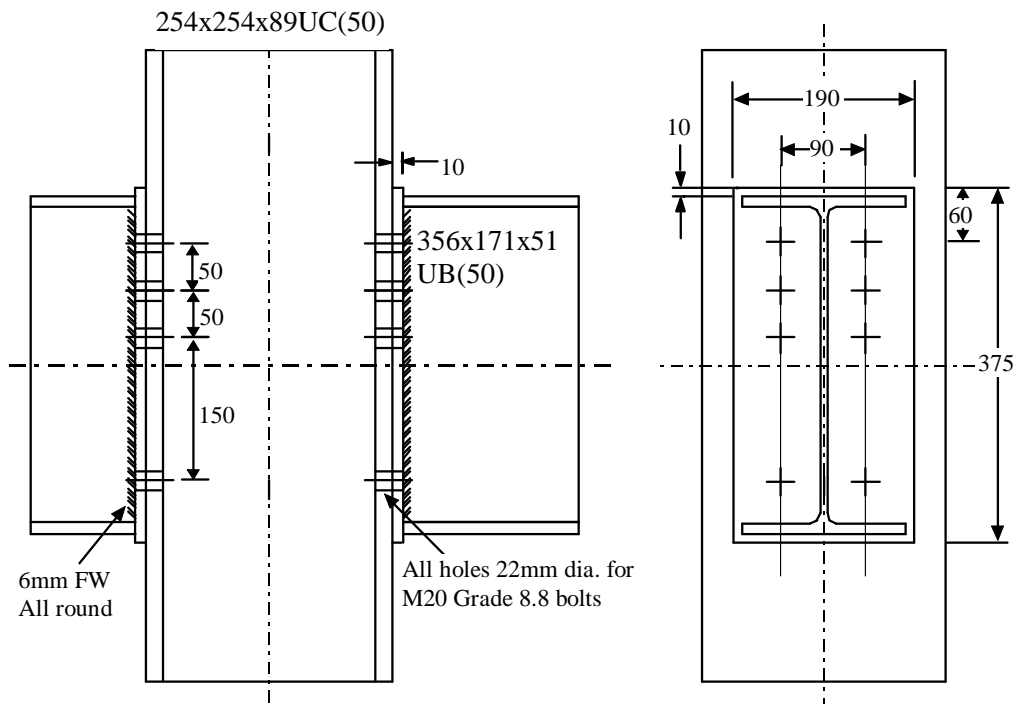

Figure 3. Flush End-Plate Joint Detail for Fire3 Tests (FR3)
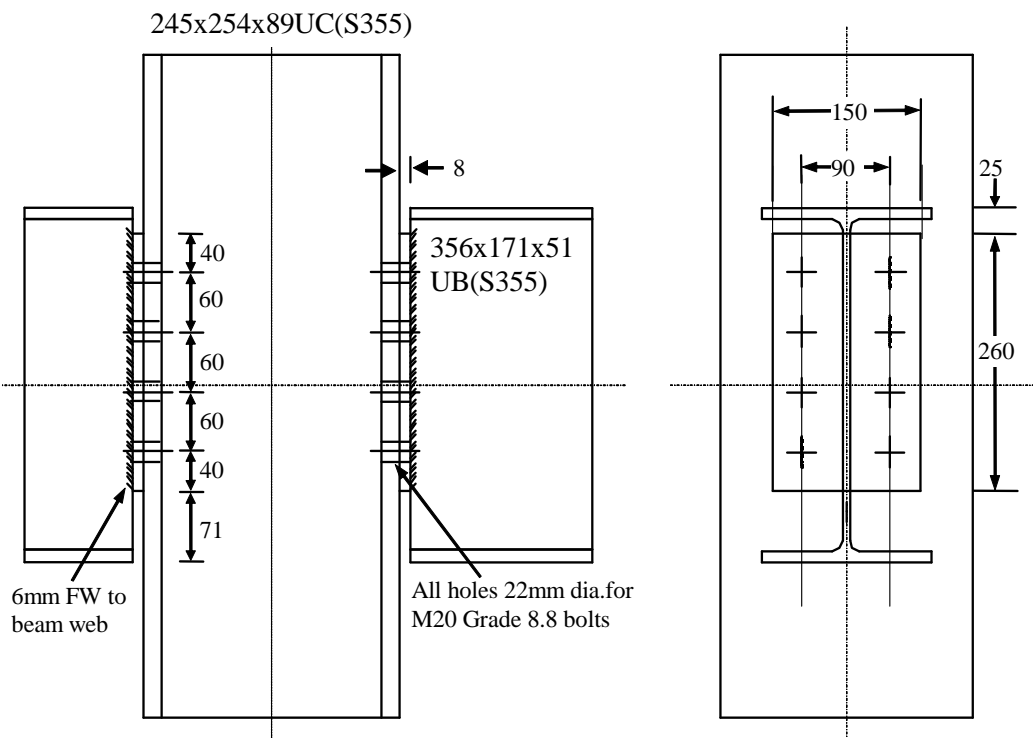

Figure 4. Flexible End-Plate Joint Detail for Fire4 Tests (FR3)

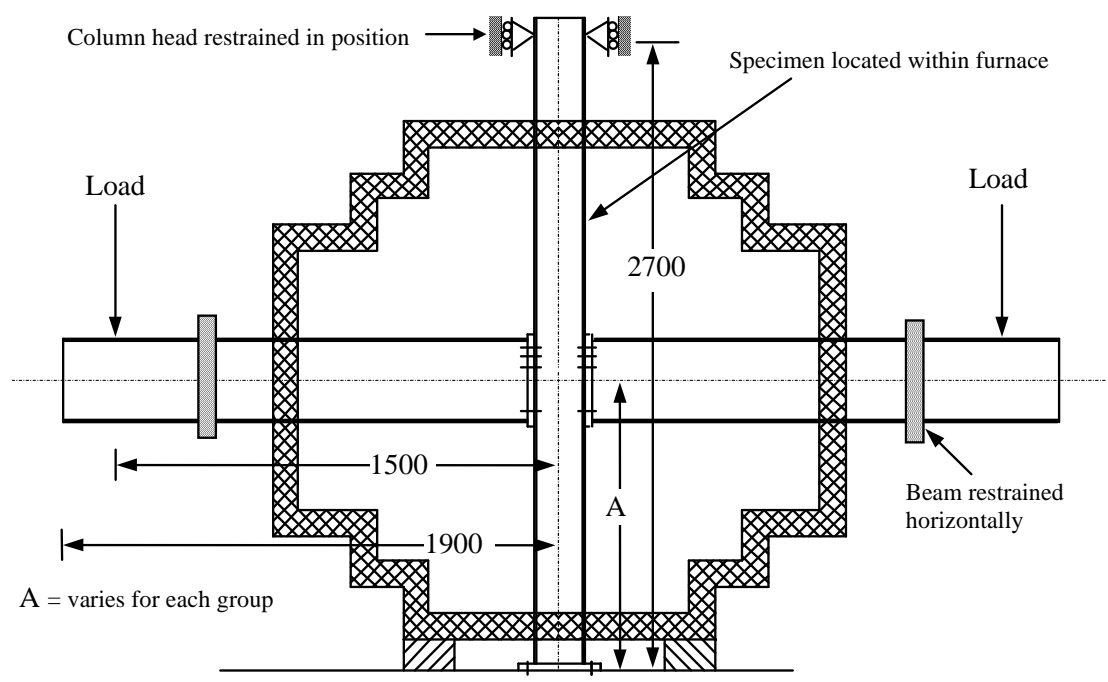

Figure 5. Elevated Temperature Testing Arrangement 
Table 1. Level of Loading for Joint Tests

\begin{tabular}{llccl}
\hline Test & & $\begin{array}{c}\text { Applied M } \\
(\mathrm{kNm}):\end{array}$ & $\begin{array}{c}\text { Average recorded M } \\
(\mathrm{kNm}):\end{array}$ & Comments: \\
\hline \multirow{5}{*}{ Fire1 } & FR11 & 4 & 4.40 & Group 1, Test 1 \\
& FR12 & 8 & 8.20 & Group 1, Test 2 \\
& FR13 & 13 & 13.12 & Group 1, Test 3 \\
& FR14 & 17 & 17.10 & Group 1, Test 4 \\
& FR21 & 5 & 4.9 & Group 2, Test 1 \\
Fire2 & FR22 & 10 & 9.3 & Group 2, Test 2 \\
& FR23 & 15 & 14.4 & Group 2, Test 3 \\
& FR24 & 20 & 19 & Group 2, Test 4 \\
& FR25 & 25 & 23 & Group 2, Test 4 \\
& FR31 & 27 & 27.4 & Group 3, Test 1 \\
Fire3 & FR32 & 56 & 54.8 & Group 3, Test 2 \\
& FR33 & 82 & 82.1 & Group 3, Test 3 \\
& FR34 & 110 & 110 & Group 3, Test 4 \\
Fire4 & FR41 & 8 & 8.2 & Group 4, Test 1 \\
& FR42 & 16 & 16.5 & Group 4, Test 2 \\
\hline
\end{tabular}

$\mathrm{M}=$ Moment applied at the joint

The fire testing arrangement consisted of a symmetric cruciform arrangement of a single column $2.7 \mathrm{~m}$ high with two cantilever beams $1.9 \mathrm{~m}$ long connected either side to the column flanges as shown in Figure 5. All specimens were major axis joints, i.e., beams connected to the column flanges with mild steel end-plates. Tests were performed in a gas-fired portable furnace lined with ceramic fibre specially designed for testing connections. A linear temperature ramp (at a rate of about $10^{\circ} \mathrm{C}$ per minute) achieving $900^{\circ} \mathrm{C}$ in 90 minutes was adopted. The instrumentation included clinometers for measuring rotations, displacement transducers, load cells and thermocouples. The experimental elevated-temperature joint tests were conducted by keeping the specimen at a constant load level and increasing the furnace temperature until failure. For all specimens, ambient-temperature material properties were measured using standard tensile coupon tests, and cross-sectional dimensions were recorded prior to testing in the furnace.

\section{DEVELOPMENT OF THE ANN MODEL}

The ANN model for semi-rigid joints was developed using the back-propagation paradigm. As shown Figure 6, 16 different input parameters were used to model the joint's moment-rotation-temperature response. These included the joint's temperature, the applied moment, material properties such as the yield strength of the joint's components and the geometry of the beams, the column, the end-plates and the bolts.

Having identified the factors that influence the rotational capacity of the joint at elevated temperature, it is necessary to establish the training samples to develop the neural network model. The experimental data were transformed into a numerical scale and coded to generate a set of training and testing data. If the rotational behaviour for a certain joint and the factors affecting this behaviour in fire are known, then it is possible to develop an empirical model for this prediction process. 


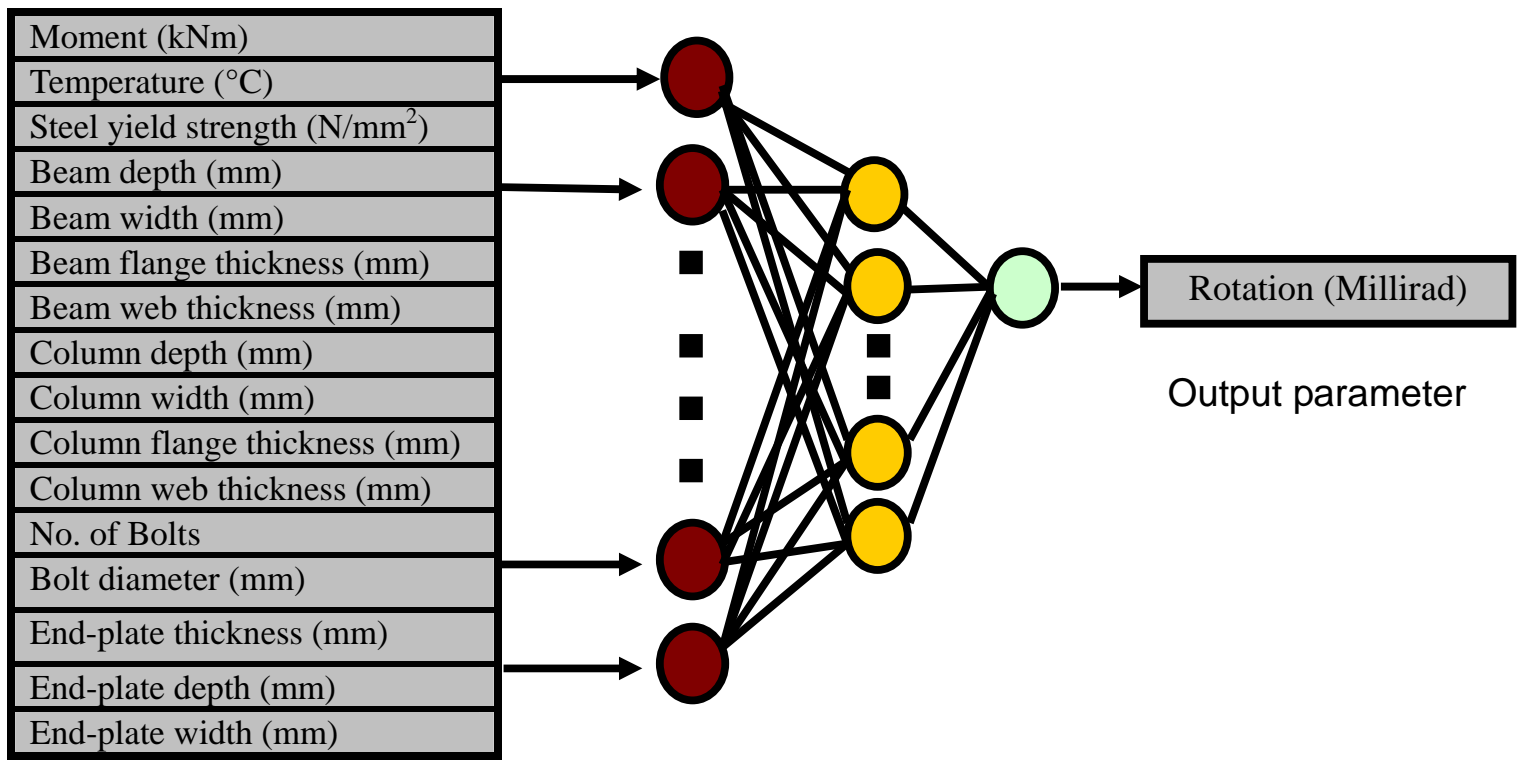

Input parameters

Figure 6. The Architecture of the Developed ANN Models

In the development of this generalized model, a training set consisting of 331 cases was utilized that were obtained from the experimental data and a testing and validation set of 61 cases that were selected randomly from the experimental data. Each training and testing data set contains variations of the 16 variables representing the different situations that could possibly take place as input and its corresponding output. Typical training patterns used as part of the training data set are shown in Table 1. The ANN model used in each case consists of 16 input nodes representing the input variables, while the output node is one representing the rotation as shown in Figure 6.

To produce a successful model, it is necessary to have a sufficient number of training cases in order to enable the ANN model to fit a continuous function to the training data. Further more, the number of training points that are needed to develop an accurate continuous model depends on other factors such as the complexity of the solution model being modelled, stochastic content of the data in which adequate training cases are required to prevent bias due to random fluctuations and the number of input variables (Al-Khaleefi et al. [6], Garson [13]).

A small learning rate of 0.4 was adopted for the current investigation since larger learning rates have been found in some cases to lead to oscillations in weight changes resulting in never ending learning process. In order to achieve an effective learning without oscillation, it is useful to make the weight change as a function of the previous weight change. This weight change is represented by the momentum coefficient. The modelling process was performed satisfactorily with a momentum coefficient value of 0.6.

The factors that provide the power of the internal representation in capturing the non-linear relationship between the input and output parameters are the number of hidden layer(s) and the number of hidden neurons in the hidden layers. Determining the number of hidden layers to use and the appropriate number of neurons to include in each hidden layer is not an exact science. Research in this area (Garson [13], Goh [14], Hecht-Nielsen [15]) proved that one or two hidden layers with an adequate number of neurons are sufficient to model any solution surface of practical interest. Therefore, a larger number of hidden layers and hidden neurons provides a good mean for developing a more effective network. However, the addition of more hidden neurons increases the number of undetermined parameters (weights and biases) associated with the network. A large 
number of training examples is then needed to solve these parameters and get a good approximation of the problem domain. When too few training examples are provided, the network will try to memorize, resulting in poor generalization (Al-Khaleefi et al. [6]). In order to provide a good approximation over the problem domain, it should be insured that the number of training pairs should be more than the number of undetermined parameters which are the weights and biases associated with the ANN approximations (Carpenter and Hoffman [16]). In this study, one hidden layer with 8 hidden neurons was found adequate to give satisfactory results.

\section{RESULTS AND DISCUSSION}

The experimental results from the fifteen different tests conducted on the four bare-steel joints were divided into a training set (331 cases) and a testing set (61 cases) which gives a total number of 392 cases that considered when developing the ANN model. The testing set (which constitutes about $15 \%$ of the data) was extracted randomly from the collected data to ensure that the developed ANN model has the capability of predicting rotational behaviour of the joints to a good degree of accuracy. The training process was performed using the NeuroShell® simulator [17]. Sixteen input neurons and one output neuron with eight hidden neurons constitute the neural network arrangement for the problem. After several adjustments to the different network parameters and after completing 12417 iterations, the network converged to a threshold of 0.0001 in less than 5 min. with a learning rate of 0.4 , a momentum value of 0.6 .

Table 2 a provides a comparison between the experimental results and the ANN generated output for some cases in the training set whereas Table $2 b$ shows a comparison between the experimental results and the ANN predicted output for selected cases in the testing set. It can be seen from Table 2 that the developed model could capture the decision process very closely. The same trend was observed in the data which is not presented in Table 2.

The trained model predictions were in good agreement with the actual experimental results as demonstrated by Figure 7 , hence, producing $\mathrm{R}^{2}$ value of 0.9639 . These results indicate that approximately more than $96 \%$ of the variation in the rotation values predicted could be explained by the 16 selected input variables and the experimental data used for model development.

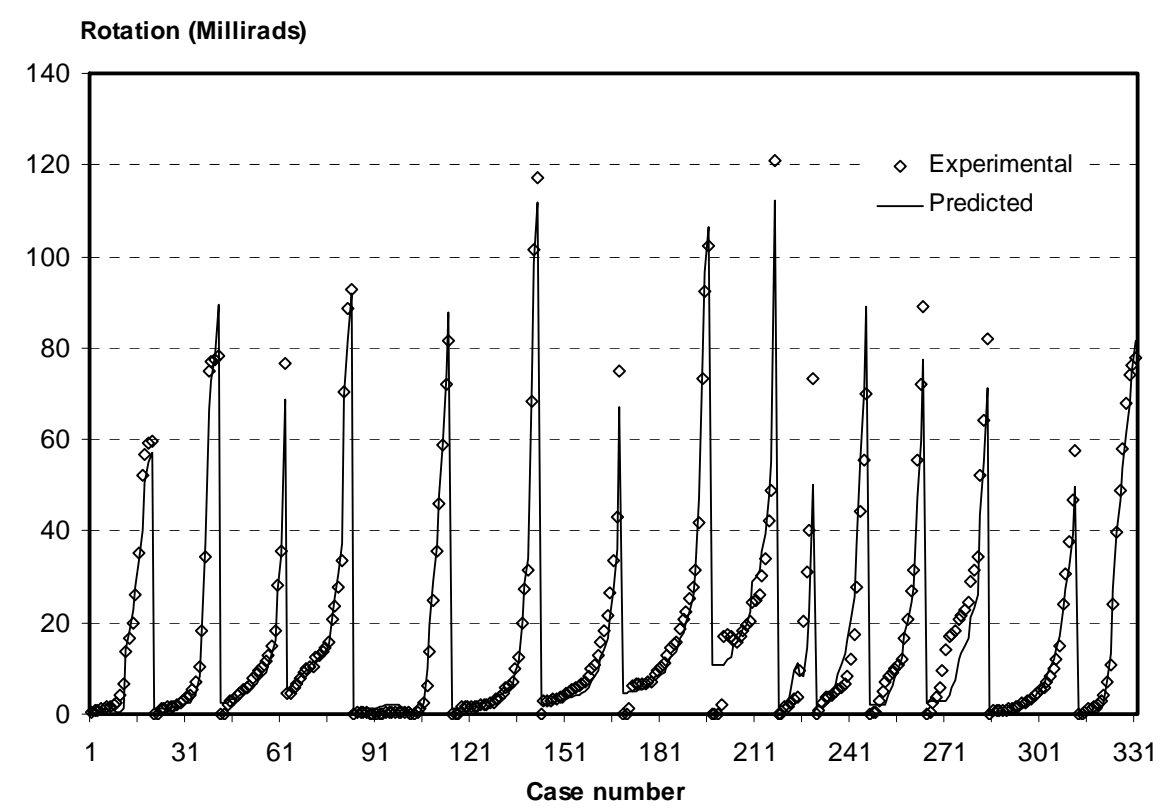

Figure 7. Comparison between Predicted and Experimental Results for the Training Set Data 


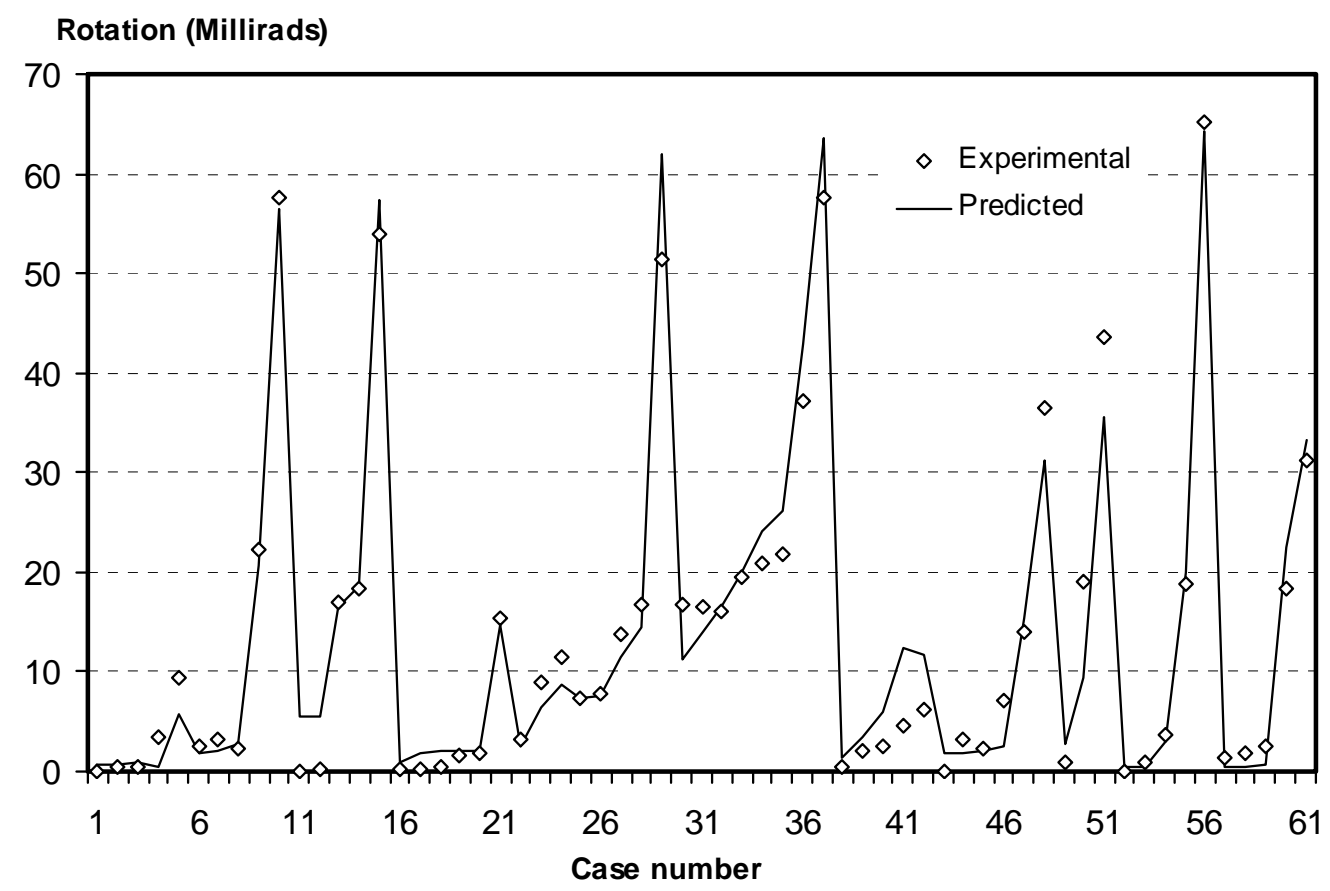

Figure 8. Comparison between Predicted and Experimental Results for the Testing Set Data

Having trained the network successfully, the next step is to test the network in order to judge its performance and to determine whether the predicted results confirm with the actual results. The ANN model can sometimes learn something different than the relationships in the data. It also can memorize the data or part of this data without learning the relationships between variables or trends in the data. Hence, to insure network accuracy and the generalization capability, the network must be tested on a continuous basis and should be monitored during the training and testing operations. The testing operation is performed by passing a separate testing set to the trained ANN model and recording the results. These results are compared to actual or experimental results. The trained model is assumed to be successful if the model gives good results for that test set or validation set. Using the testing and validation set of 61 cases that were extracted randomly from the experimental data for the model; the testing operations were then performed on the model separately. The results were compared with the actual experimental results. The statistical analysis of these results indicates that the $\mathrm{R}^{2}$ value for the testing set was 0.9562 as shown in Figure 8 . This high generalization capability indicates that the ANN model developed in this work can be used to model and predict the relationship accurately based on the given input variables. However it should be noted from Figures. 7 and 8 that at very low rotations the developed ANN predicting higher rotations that the experimental ones while at very large rotational levels lower values of rotation was predicted compared to experimental results. This variation was observed in both training and testing sets respectively. This discrepancy between the experimental and predicted rotations will not affect the reliability of the model since practically the actual behaviour of the joint can rarely undergo very large levels of rotation beyond the levels closely predicted by the model even at very high temperatures. 
Table 2. Comparison between Predicted Results from the Neural Network Model Generated vs. Experimental Rotations on Some Training and Testing Cases

\begin{tabular}{|c|c|c|}
\hline Case \# & Experimental rotation & ANN generated rotation \\
\hline \multicolumn{3}{|c|}{ (a) training cases } \\
\hline 2 & 0.71 & 0.92 \\
\hline 15 & 26.22 & 27.92 \\
\hline 20 & 59.55 & 57.25 \\
\hline 35 & 10.22 & 8.33 \\
\hline 52 & 8.05 & 6.17 \\
\hline 59 & 18.33 & 16.28 \\
\hline 73 & 12.98 & 12.28 \\
\hline 80 & 33.49 & 37.32 \\
\hline 89 & 0.12 & 1.23 \\
\hline 109 & 24.84 & 28.98 \\
\hline 122 & 1.55 & 1.62 \\
\hline 142 & 117.1 & 111.6 \\
\hline 161 & 12.71 & 9.58 \\
\hline 186 & 15.9 & 13.79 \\
\hline 201 & 16.83 & 10.65 \\
\hline 225 & 9.36 & 8.57 \\
\hline 246 & 70.08 & 88.86 \\
\hline 288 & 0.75 & 0.41 \\
\hline 300 & 4.52 & 4.46 \\
\hline 312 & 57.66 & 49.76 \\
\hline 325 & 39.72 & 39.79 \\
\hline 331 & 77.89 & 81.43 \\
\hline \multicolumn{3}{|c|}{ (b) testing cases } \\
\hline 3 & 0.38 & 0.83 \\
\hline 7 & 3.21 & 2.16 \\
\hline 10 & 57.54 & 56.39 \\
\hline 16 & 0.17 & 0.83 \\
\hline 21 & 15.36 & 14.65 \\
\hline 27 & 13.71 & 11.53 \\
\hline 32 & 16.01 & 16.5 \\
\hline 36 & 37.26 & 42.85 \\
\hline 41 & 4.62 & 12.4 \\
\hline 48 & 36.4 & 31.3 \\
\hline 55 & 18.84 & 19.88 \\
\hline 61 & 31.17 & 33.37 \\
\hline
\end{tabular}

Table 3. Percent Contribution of the Input Variables to the Rotation in the Three Models

\begin{tabular}{|llcc}
\hline \multicolumn{1}{c}{ Joint's Component } & \multicolumn{1}{c}{ Input parameter } & \% Contribution & RANK \\
\hline & Moment $(\mathrm{kNm})$ & 33.04 & 1 \\
& Temperature $\left({ }^{\circ} \mathrm{C}\right)$ & 28.65 & 2 \\
\multirow{3}{*}{ Beam } & Depth & 1.47 & 16 \\
& Width & 1.73 & 14 \\
& Flange thickness & 1.73 & 13 \\
& Web thickness & 1.80 & 10 \\
Column & Depth & 1.82 & 8 \\
& Width & 2.02 & 6 \\
& Flange thickness & 1.80 & 11 \\
Bolts & Web thickness & 1.87 & 9 \\
& No. of Bolts & 1.55 & 15 \\
End-plate & Bolt diameter & 1.92 & 7 \\
& Thickness & 3.58 & 5 \\
& Depth & 5.92 & 4 \\
& Width & 9.35 & 3 \\
& Steel yield strength & 1.75 & 12 \\
\hline
\end{tabular}


To find the percent contribution of each of the input variables with respect to the output variables, the partitioning method of the connection weights of the network (Garson [13], Goh [14]) was used. It was found that the highest contribution is due to the moment (33.04\%), temperature (28.65\%), and end-plate width (9.35\%), depth (5.92\%), and thickness (3.58\%). These results, as shown in Table 3, clearly indicate that the temperature, the moment and the geometries of the end-plate are important factors in determining the effect elevated temperature on the degree of rotation.

\section{CONCLUSIONS}

This paper presented an artificial neural network (ANN) model developed to predict the rotational behaviour of semi-rigid joints in fire. Data from three flush end-plate and flexible end-plate bare-steel joints were modelled and results from fifteen different tests were evaluated. Data from these tests were used for training and testing the neural network model. The results obtained from the model compared very closely with the experimental results with $\mathrm{R}^{2}$ value of 0.964 and 0.956 for training and testing sets, respectively demonstrating the capability of the ANN simulation techniques in predicting the behaviour of semi-rigid joints in fire with high accuracy. Results also showed that the applied moment, temperature and end-plate geometry have considerable effect on the rotation of joints in fire more than the other input parameters studied. The described model can be modified to study other important parameters that can have considerable effect on the behaviour of joints at elevated temperatures.

\section{REFERENCES}

[1] Al-Jabri, K.S., Burgess, I.W., Lennon, T. and Plank, R.J., "Moment-Rotation-Temperature Curves for Semi-Rigid Joints”, Journal of Constructional Steel Research, 2005, Vol. 61, pp. 281-303.

[2] Stavroulakis, G.E. and Abdalla, K.M., “A Systematic Neural Network Classificator in Mechanics. An Application in Semi-rigid Steel Joints”, International Journal for Engineering Analysis and Design, 1994, Vol. 1, pp. 279-292.

[3] Stavroulakis, G.E., Avdelas, A.V., Abdalla, K.M. and Panagiotopoulos, P.D., "A Neural Network Approach to the Modelling, Calculation and Identification of Semi-rigid Connections in Steel Structures”, Journal of Constructional Steel Research, 1997, Vol. 44, No. 1-2, pp. 91-105.

[4] Anderson, D., Hines, E.L., Arthur, S.J. and Eiap, E.L., “Application of Artificial Neural Networks to the Prediction of Minor Axis Steel Connections", Computers and Structures, 1997, Vol. 63, No. 4, pp. 685-692.

[5] Dermitas, B., De Santiago, E. and O'leary, J.R., "Classification of Steel Semi-rigid Connections by Neural Networks”, Structures 2004-Building on the Past: Securing the future, 2004. Nashville, Tennessee, USA.

[6] Al-Khaleefi, A.M., Terro, M.J., Alex, A.P. and Wang, Y., "Prediction of Fire Resistance of Concrete Filled Tubular Steel Columns using Neural Networks”, Fire Safety Journal, 2002, Vol. 37, pp. 339-352.

[7] Rumelhart, D. and McClelland, J., "PDP Research Group. Parallel Distributed Processing, Explorations in the Microstructure of Cognition, Vol. 11: Foundations”, Cambridge, MA, MIT Press/Bradford Books, 1988.

[8] Stanley, J., "Introduction to Neural Networks", $3^{\text {rd }}$ Edition, Sierra Madre, California Scientific Software, California, 1990.

[9] Simpson, P.K., “Artificial Neural Systems: Foundations, Paradigms, Applications, and Implementations”, $1^{\text {st }}$ Edition, Elmsford, NY, Pergamon Press, Inc., 1990. 
[10] Guzelbey, I.H., Cevik, A. and Gögüs, M.T., "Prediction of Rotation Capacity of Wide Flange Beams using Neural Networks”, Journal of Constructional Steel Research, 2006, Vol. 62, pp. 950-961.

[11] Guzelbey, I.H., Cevik, A., and Erklig, A., "Prediction of Web Crippling of Cold-Formed Steel Sheetings using Neural Networks", Journal of Constructional Steel Research, 2006; Vol. 62, pp. 962-973.

[12] Leston-Jones, L.C., "The Influence of Semi-rigid Connections on the Performance of Steel Framed Structures in Fire”, PhD, Thesis, University of Sheffield, UK, 1997.

[13] Garson, G.D., “Interpreting Neural Network Connection Weights”, AI Expert; 1991, Vol. 6, No. 7, pp. 47-51.

[14] Goh, A.T.C., "Back-Propagation Neural Networks for Modelling Complex Systems", Artificial Intelligence in Engineering, 1995, Vol. 9, pp. 143-151.

[15] Hecht-Nielsen, P., "Theory of the Back Propagation Neural Network", Proceeding of International Conference on Neural Networks. Washington D.C., 1989, pp. 593-605.

[16] Carpenter, W.C. and Hoffman, M.E., "Training Back Prop Neural Networks, AI Expert, 1995, Vol. 10, pp. 30-33.

[17] NeuroShell ${ }^{\mathrm{TM}}$., "Neural Network Shell Program”, $4^{\text {th }}$ Edition. Ward Systems Group, Inc., Frederick, MD, USA, 1991. 